Entwicklungszusammenarbeit und Wissenstransfer

\section{Umweltrechnungslegung in Südostasien}

Die Umweltrechnungslegung unterstützt die Integration

ökologischer und ökonomischer Informationen für

Entscheidungsprozesse. Ein internationales Forschungs-, Transfer- und Weiterbildungsprojekt verankert dieses

Konzept im südostasiatischen Raum. Von Christian Herzig,

Stefan Schaltegger, Günter Tharun und Tobias Viere

$\mathrm{E}_{\mathrm{d}}^{\mathrm{in}}$ n steigender Anteil der globalen Industrieproduktion findet in Südostasien statt. Die Region zeichnet sich durch ein hohes Wirtschaftswachstum aus, das zu entsprechend steigenden Umweltproblemen führt. In solchen Regionen besitzt das Umweltrechnungswesen (Englisch: Environmental Management Accounting, EMA) ein besonders hohes Potenzial, denn nur wenn Entscheidungsträger über Umweltaspekte richtig informiert sind, können sie systematisch berücksichtigt werden.

Die Instrumente der betrieblichen Umweltrechnungslegung versorgen die Entscheidungsträger eines Unternehmens mit für sie relevanten, umweltbezogenen Informationen. Diese Informationen können sowohl monetärer als auch nichtmonetärer Natur sein und werden für eine Vielzahl betrieblicher Entscheidungssituationen herangezogen (Burritt et al. 2002). Dabei kommen situationsspezifisch unterschiedliche EMAInstrumente zum Einsatz, zum Beispiel Umweltkostenrechnungen, Stoffstromanalysen, Umweltinvestitionsrechnungen oder Umweltbudgetierungen.

\section{Konzept mit Potenzial}

Aufgrund der situations- und praxisbezogenen Aufbereitung und Integration ökonomischer und ökologischer Informationen hat EMA das Potenzial, ein wichtiges, wertschaffendes Konzept für eine nachhaltige Unternehmens- und Geschäftsentwicklung zu werden. Obwohl
EMA in seiner Gesamtheit ein relativ neues Konzept darstellt, haben einzelne Instrumente wie die Flusskostenrechnung in einigen industrialisierten Ländern bereits weite Verbreitung gefunden. In sich gerade industrialisierenden Ländern gibt es hingegen wenige Erfahrungen mit der systematischen Generierung und Anwendung von umweltbezogenen Informationen in Unternehmen und ihrer erfolgsrelevanten Aufbereitung und Verknüpfung mit ökonomischen Informationen.

Vor diesem Hintergrund hat sich die Internationale Weiterbildung und Entwicklung gGmbH (InWEnt) im Jahr 2003 entschlossen, das Projekt Umweltrechnungslegung für kleine und mittelständische Unternehmen im südostasiatischen Raum ins Leben zu rufen. Das vom Bundesministerium für wirtschaftliche Zusammenarbeit und Entwicklung geförderte Projekt verfolgt das Ziel, das EMAKonzept in den Ländern Indonesien, Thailand, Vietnam und auf den Philippinen $\mathrm{zu}$ verbreiten.

Hierzu führt das Centre for Sustainability Management (CSM) der Universität Lüneburg in Kooperation mit asiatischen Partnerorganisationen bis Ende 2007 Unternehmensfallstudien, Weiterbildungsseminare, Training of Trainers und weitere Maßnahmen für den Know-how Transfer besonders für kleine und mittelständische Unternehmen durch. Dabei werden die besonderen Anforderungen kleiner und mittelständischer Unternehmen im Umwelt- und Entwicklungszusammenarbeitskontext berücksichtigt.
Die einzelnen Maßnahmen, die das Projekt als ein Forschungs-, Transfer- und Weiterbildungsprojekt kennzeichnen, werden im Folgenden näher erläutert.

\section{Initialisierung und Vernetzung}

Die Durchführung von 16 Informationsworkshops in den vier Zielländern diente der Bekanntmachung des EMASEA-Projekts sowie des im südostasiatischen Raum bislang wenig verbreiteten Themas der Umweltrechnungslegung. Hierzu wurden vom asiatischen Hauptprojektpartner Asian Society for Environmental Protection (ASEP) sowohl Manager von Unternehmen als auch Vertreter von Beratungsunternehmen, Behörden, Nichtregierungsorganisationen, Vereinen und Verbänden sowie Forschungseinrichtungen eingeladen. Zugleich wurden erste Schritte zur institutionellen Vernetzung, Partnerschafts- und Netzwerkbildung eingeleitet. Eine Reihe von Unternehmen haben sich daraufhin um die Durchführung von EMA-Fallstudien beworben. Einzelne von ihnen wurden für die Forschungsstudie des Projekts ausgewählt.

Eine Forschungsstudie untersucht die Links zwischen EMA-Instrumenten und ihren Anwendern im Kontext betrieblicher Entscheidungssituationen. Hierbei handelt es sich um eine vergleichende Fallstudie, die 16 explorative Einzelfallstudien in kleinen und mittleren Unternehmen der vier Zielländer umfasst (Herzig et al. im Erscheinen).

Damit unterscheidet sich das Forschungsvorhaben von der Mehrzahl bisheriger EMA-Fallstudien, die insbesondere die Anwendung von spezifischen Einzelinstrumenten zum Ziel haben und dabei vorwiegend auf die Implementation und die Kosten-/Nutzenbetrachtung des Einzelinstrumenteneinsatzes fokussieren. Die EMA-SEA-Forschungsstudie betrachtet EMA hingegen aus einer anderen Perspektive: Sie hat das Ziel, die komplexen Entscheidungssituationen in Unternehmen zu verstehen und Unterschiede sowie Gemeinsamkeiten von Entscheidungsprozessen herauszuarbeiten. 
In Zusammenarbeit mit den Praxisakteuren werden die spezifischen Problemstellungen, Entscheidungssituationen und Rechnungslegungsprozesse analysiert und aufgezeigt, inwieweit durch den Einsatz unterschiedlicher EMA-Instrumente die Integration von Umweltaspekten in die unternehmerischen Prozesse gefördert werden kann. Die Ergebnisse der Fallstudienanalyse sollen die Anwendbarkeit des EMA-Rahmenkonzepts verdeutlichen sowie gegebenenfalls eine Basis seiner Fortentwicklung bieten. Darüber hinaus fokussiert die transdisziplinäre Fallstudie auf kleine und mittlere Unternehmen in sich industrialisierenden Ländern, die bislang in der Fallstudienforschung zu EMA nachrangig behandelt wurden.

\section{Weiterbildung zu EMA}

Aufbauend auf den Fallstudien werden praxisorientierte Trainingsmaterialien entwickelt und in verschiedenen Weiterbildungsseminaren und -kursen eingesetzt. Das Weiterbildungsprogramm umfasst Präsenzseminare, bei denen die Vermittlung von handlungsorientiertem Wissen bezüglich EMA und der wichtigsten EMA-Instrumente im Mittelpunkt stehen. Diese von ASEP organisierten Seminare basieren auf einer aktiven Trainingsmethode, dem Project Case Work Approach (Tharun 2001). Sie beinhalten interaktive rollenbasierte Gruppenarbeiten, die mit einer projektorientierten Szenariomethode verknüpft sind. Die Schulung wird auch als Online-Kurs angeboten, um Manager mit Zeit- und Entfernungsproblemen erreichen zu können.

Auf diesem Kurs aufbauend werden ausgewählte Teilnehmer zu EMA-Trainern für ihr jeweiliges Heimatland ausgebildet. Hierzu setzen sich die zukünftigen Multiplikatoren im Rahmen einer Online-Schulung intensiv mit weiteren EMA-Instrumenten und unternehmensspezifischen Entscheidungssituationen auseinander, wobei Lehrfallstudien die Grundlage für ein szenariobasiertes und problembezogenes Lernen an Realsituationen bilden. Im Anschluss daran nehmen die zukünftigen Trainer an einem
Kursleitertraining teil, dem so genannten Training of Trainers (TOT). Ziel ist es, die Trainer zu einer selbständigen Durchführung von EMA-Seminaren und -Unternehmensprojekten zu befähigen. Sie sollen zum einen in der Lage sein, in Zusammenarbeit mit Managern eines Unternehmens die jeweilige Entscheidungs- und Problemsituationen zu analysieren und mit Hilfe geeigneter EMAInstrumente bewältigen $\mathrm{zu}$ können. Zum anderen werden die Trainer ausgebildet, eigene Seminare durchzuführen und EMA-bezogenes Wissen zu vermitteln.

\section{Wissenstransfer und Verbreitung}

Die Multiplikatorenausbildung bildet den Ausgangspunkt für den weiteren Wissenstransfer im südostasiatischen Wirtschaftsraum. Ausgebildete EMA-Trainer werden zu Beginn bei der eigenen Durchführung von EMA-Seminaren und Unternehmensprojekten von CSM und ASEP begleitet, um die Qualität der Trainingsmaßnahmen sowie eine erfolgreiche EMA-Implementierung in Unternehmen sicherzustellen. Mittel- bis langfristig wird dadurch ein sich selbst tragender Prozess der Verbreitung und Anwendung von EMA in Südostasien angestrebt. Ein Netzwerk aus Fachexperten, unternehmensnahen Organisationen (z.B. Industrie- und Handelskammern), Universitäten, NGOs und den Unternehmen selbst soll eine langfristige Verbreitung und Umsetzung von EMA im südostasiatischen Raum über das Projektende hinaus sicherstellen (Viere et al. im Erscheinen).

\section{Ausblick}

Erste Ergebnisse der durchgeführten Unternehmensfallstudien zeigen, dass das Potenzial der Umweltrechnungslegung in industrialisierenden Ländern besonders groß ist. Die Verbreitung des EMA-Konzepts erfordert eine partnerschaftliche Kooperation aller beteiligten Individuen und Organisationen. Durch das Zusammenwirken von Forschung, Wissenstransfer und Weiterbildung kann sich dann ein selbst tragendes Netzwerk von EMA-Akteuren in Südostasien entwickeln.

\section{Literatur}

Burritt, R.; Hahn, T.; Schaltegger, S.: Towards a Comprehensive Framework for Environmental Management Accounting. Links between Business Actors and Environmental Management Accounting Tools. In: Australian Accounting Review 12(2) 2002, S. 39-50.

Herzig, C.; Viere, T.; Burritt, R.; Schaltegger, S.: „Understanding and supporting management decision-making: South-East Asian case studies on Environmental Management Accounting". In: Schaltegger, Stefan; Bennett, Martin; Bouma, Jan Jaap \& Burritt, Roger (Hrsg.): Sustainability Accounting and Reporting. Berlin, im Erscheinen.

Tharun, G.: The Project Casework (PCW) Concept in Brief. Its Rationale, Structure, Function, Development and Origin in 42 Statements. Original Version 1984.

Viere, T.; Herzig, C.; Schaltegger, S; Leung, R.: Partnerships for corporate sustainability. Capacity development in South-East Asia In: Welford, Richard (Hrsg.): Partnerships for Sustainable Development: Perspectives from the Asia-Pacific Region, im Erscheinen.

\section{AUTORINNEN + KONTAKT}

Prof. Dr. Stefan Schaltegger ist Inhaber der Professur für BWL, insb. Umweltmanagement, und Leiter des Centre for Sustainability Management der Universität Lüneburg.

Christian Herzig und Tobias Viere sind Doktoranden und wissenschaftliche Mitarbeiter am selben Institut.

Centre for Sustainability Management, Universität Lüneburg, 21332 Lüneburg. E-Mail: ema-sea@uni-lueneburg.de. Projektwebsite:

www.environmental-accounting.org

Dr. Günter Tharun ist Projektleiter bei der InWEnt - Internationale Weiterbildung und Entwicklung gGmbH, Köln.

InWEnt gGmbH, Abt. 405 Nachhaltige Technologie, Industrieund Stadtentwicklung. Weyerstraße 79-83, 50676 Köln. Tel. 0221/ $2098-0$

E-Mail: guenter.tharun@inwent.org 
(c) 20I0 Authors; licensee IÖW and oekom verlag. This is an article distributed under the terms of the Creative Commons Attribution Non-Commercial No Derivates License (http://creativecommons.org/licenses/by-nc-nd/3.o/), which permits unrestricted use, distribution, and reproduction in any medium, provided the original work is properly cited. 